\title{
Analysis of Wet Pressing of Paper: The Three-Phase Model. Part II: Compressible Air Case
}

\author{
D. BEŽANOVIĆ* , C. J. VAN DUIJN and E. F. KAASSCHIETER \\ Department of Mathematics and Computer Science, Eindhoven University of Technology, \\ P.O. Box 513, 5600 MB Eindhoven, The Netherlands
}

(Received: 8 July 2005; accepted in final form: 1 February 2006)

\begin{abstract}
In this study we consider a model of wet pressing of paper. We use the techniques and results from the first part of this paper, where a simplified model is studied in details. The model is, using suitable transformation, rewritten in the standard parabolic-hyperbolic form. Numerical solution for typical example is given and the effects of plastic deformations of paper are investigated. Finally, the model is employed to adres the problem of choosing an optimal pressing regime.
\end{abstract}

Key words: paper pressing, compressible air, parabolic-hyperbolic system, cross conditions, elastic and plastic deformations, upwind method.

\section{Introduction}

In Part I (Bežanović et al., 2007) we outlined under the simplified conditions of constant air density some characteristics of the wet pressing process, involving two-phase flow in deformable porous media. In this second part we investigate a more practical setting. More precisely, we consider compressible air and prescribe 'no-flow' boundary conditions. In Part I we considered two possible numerical approaches: saturation upwind (s-upwind) and front tracking. The emphasize in this part is on the numerical treatment of the extended model. For this purpose we use the s-upwind scheme introduced in Part I. We will also make comparisons with other models and experiments.

This paper is organized as follows. In Section 2 we recall the equations for the extended model including initial, boundary and the cross conditions. The most important analytical features of the model are explained in Section 3. In Section 4 we first give a typical numerical example. Then we include the effect of permanent (plastic) deformation of paper and we 
finally employ the model to address the problem of the improving the efficiency of press-nip. The conclusions are given in Section 5.

\section{Mathematical Model}

Most of the assumptions are the same as in Part I. Paper and felt are considered as deformable porous media and only transversal flow and deformations are considered. The mechanical response of the solid structures of the layers is, for the time being, considered to be perfectly elastic. Water and solid phase are considered to be intrinsically incompressible.

Opposite to the previous case, the compression of air is now taken into account. Air is assumed to obey a perfect gas equation:

$$
p_{\mathrm{a}}=p_{\mathrm{a} 0}+\gamma\left(\rho_{\mathrm{a}}-\rho_{\mathrm{a} 0}\right),
$$

where $p_{\mathrm{a} 0}$ and $\rho_{\mathrm{a} 0}$ are the pressure and the density of air at atmospheric conditions and $\gamma$ is a known positive constant. For an arbitrary material control volume $V=\left(Z_{1}, Z_{2}\right)$ (see for more details Part I) the mass balance of air in integral form reads

$$
\frac{\mathrm{d}}{\mathrm{d} t} \int_{V} \phi(1+\epsilon)(1-s) \rho_{\mathrm{a}} \mathrm{d} Z-\rho_{\mathrm{a}} q_{\mathrm{a}}\left(Z_{1}, t\right)+\rho_{\mathrm{a}} q_{\mathrm{a}}\left(Z_{2}, t\right)=0 .
$$

Here $\phi$ denotes porosity, $s$ saturation, $\epsilon$ strain, while $q_{\mathrm{a}}$ is the specific air discharge, relative to the solid phase (for more details see again Part I). Instead of the transversal spatial coordinate $z$, the corresponding material coordinate $Z$ is used. Disregarding capillary effects in pores we write

$$
p_{\mathrm{a}}=p_{\mathrm{w}}=p_{\mathrm{f}},
$$

and we refer to $p_{\mathrm{f}}$ as the fluid pressure. With this assumption, the total applied pressure $p_{\mathrm{T}}$ is divided over solid and fluid phases accordingly to Terzaghi's principle (Bear, 1972), implying

$$
p_{\mathrm{T}}=p_{\mathrm{s}}+p_{\mathrm{f}},
$$

where $p_{\mathrm{s}}$ is the effective structural pressure. We assume that the total pressure is given as a function of time, $p_{\mathrm{T}}=p_{\mathrm{T}}(t)$. The elastic response of the solid skeleton of the layers is modelled by a functional relationship between structural pressure and strain. As in Part I, this relation can be rewritten in terms of structural pressure and void ratio, yielding

$$
p_{\mathrm{s}}=p_{\mathrm{s}}(u)=p_{\mathrm{s} 0}\left(u^{-q}-u_{0}^{-q}\right), \quad q>0 .
$$

Terzaghi's principle (2), together with (1) and (3), gives

$$
\rho_{\mathrm{a}}=\rho_{\mathrm{a}}(u, t)=\rho_{\mathrm{a} 0}+\frac{1}{\gamma}\left[p_{\mathrm{T}}(t)-p_{\mathrm{s} 0}\left(u^{-q}-u_{0}^{-q}\right)-p_{\mathrm{a} 0}\right] .
$$


Here, the so-called scaled void ratio $u$ is given by

$$
u=\left(1-\phi_{0}\right) \frac{\phi}{1-\phi},
$$

where $\phi_{0}=u_{0}$ is the value of $\phi$ that corresponds to undeformed state. We use Darcy's law in material coordinates

$$
q_{j}=-\frac{k(u) k_{j}^{r}(s)}{\mu_{j}(1+\epsilon)} \frac{\partial p_{j}}{\partial Z}, \quad j=\mathrm{w}, \mathrm{a},
$$

where $\mu_{j}$ is viscosity, $k$ intrinsic permeability and $k_{j}^{\mathrm{r}}$ relative permeability of phase $j, j=\mathrm{w}$, a (subscripts $\mathrm{w}$ and a refer to water and air, respectively). The following functional dependances are used, see (Bear, 1972; Helmig, 1997):

$$
k=k(u)=k_{0} \frac{u^{3}}{\left(1-\phi_{0}\right)^{2}\left(1-\phi_{0}+u\right)} \quad(\text { Kozeny-Carman }),
$$

and

$$
\begin{aligned}
& k_{\mathrm{w}}^{\mathrm{r}}(s)=s^{\frac{2+3 \lambda}{\lambda}}, \\
& k_{\mathrm{a}}^{\mathrm{r}}(s)=(1-s)^{2}\left(1-s^{\frac{2+\lambda}{\lambda}}\right), \quad 0.2<\lambda<3 \quad \text { (Brooks-Corey). }
\end{aligned}
$$

Analogously to Part I we introduce the dimensionless coordinates

$$
x=\frac{Z}{h_{0}} \text { and } t=\frac{t}{t_{\text {fin }}},
$$

where $h_{0}$ is the total initial thickness of the paper-felt system and $t_{\text {fin }}$ is the total time of the press-nip. Furthermore, we introduce the dimensionless pressures and air density by redefining

$$
p_{\mathrm{s}}=\frac{p_{\mathrm{s}}}{p_{\mathrm{s} 0}}, \quad p_{\mathrm{f}}=\frac{p_{\mathrm{f}}}{p_{\mathrm{s} 0}}, \quad p_{\mathrm{T}}=\frac{p_{\mathrm{T}}}{p_{\mathrm{s} 0}} \quad \text { and } \quad \rho_{\mathrm{a}}=\frac{\rho_{\mathrm{a}}}{\rho_{\mathrm{a} 0}} .
$$

With this change, (1), (3) and (4) become, respectively

$$
\begin{aligned}
& p_{\mathrm{f}}=\frac{p_{\mathrm{a} 0}}{p_{\mathrm{s} 0}}+\gamma^{*}\left(\rho_{\mathrm{a}}-1\right), \\
& p_{\mathrm{s}}(u)=u^{-q}-u_{0}^{-q},
\end{aligned}
$$

and

$$
\rho_{\mathrm{a}}(u, t)=1+\frac{1}{\gamma^{*}}\left[p_{\mathrm{T}}(t)-\left(u^{-q}-u_{0}^{-q}\right)-\frac{p_{\mathrm{a} 0}}{p_{\mathrm{s} 0}}\right],
$$

for a dimensionless quantity

$$
\gamma^{*}=\gamma \frac{\rho_{\mathrm{a} 0}}{p_{\mathrm{s} 0}}
$$


The final system now reads

$$
(u s)_{t}=\left(C_{\mathrm{w}}(u, s) u_{x}\right)_{x} \quad \text { (water equation), }
$$

and

$$
\left(u(1-s) \rho_{\mathrm{a}}(u, t)\right)_{\mathrm{t}}=\left(\rho_{\mathrm{a}}(u, t) C_{\mathrm{a}}(u, s) u_{x}\right)_{x} \quad \text { (air equation) . }
$$

Here the indices $x$ and $t$ denote partial differentiation with respect to these variables. The functions $C_{j}, j=\mathrm{w}$, a are given by

$$
\begin{aligned}
C_{j}(u, s) & =-\frac{t_{\text {fin }} p_{\mathrm{s} 0}}{h_{0}^{2}} \frac{k(u) k_{j}^{\mathrm{r}}(s)}{\mu_{j}(1+\epsilon(u))} \frac{d p_{\mathrm{s}}}{d u} \\
& =\frac{q t_{\mathrm{fin}} k_{0} p_{\mathrm{s} 0}}{h_{0}^{2} \mu_{j}\left(1-\phi_{0}\right)^{2}} \frac{u^{2-q} k_{j}^{\mathrm{r}}(s)}{\left(1-\phi_{0}+u\right)^{2}}=C_{j 0} g(u) k_{j}^{\mathrm{r}}(s) .
\end{aligned}
$$

Equations (8) and (9) hold in both the paper and the felt domain:

paper: $Q^{\mathrm{p}}=\left\{(x, t): 0<x<x_{c}, 0<t<1\right\}$,

felt: $Q^{\mathrm{f}}=\left\{(x, t): x_{c}<x<1,0<t<1\right\}$.

Here $x_{c}=h_{0 \mathrm{p}} / h_{0}$ corresponds to the paper-felt interface. The values of the constants in equations (8) and (9), when considered in the subdomains $Q^{\mathrm{p}}$ and $Q^{\mathrm{f}}$, are denoted by the superscript $i$. Thus we have $C_{j 0}^{i}, \phi_{0}^{i}, q^{i}, g^{i}(u)$ and $k_{j}^{\mathrm{ri}}(s)$, with $j=\mathrm{w}$, a and $i=\mathrm{p}, \mathrm{f}$.

\subsection{INITIAL, BOUNDARY AND CROSS CONDITIONS}

When solving equations (8) and (9) in the paper $\left(Q^{\mathrm{p}}\right)$ and the felt $\left(Q^{\mathrm{f}}\right)$ one needs initial, boundary, and cross conditions between them.

\subsubsection{Initial condition}

The same initial conditions as in Part I are prescribed. Layers are initially undeformed, giving

$$
u(x, 0)=\left\{\begin{array}{lll}
u_{0}^{\mathrm{p}}=\phi_{0}^{\mathrm{p}} & \text { for } & x \in\left(0, x_{c}\right), \\
u_{0}^{\mathrm{f}}=\phi_{0}^{\mathrm{f}} & \text { for } & x \in\left(x_{c}, 1\right) .
\end{array}\right.
$$

Furthermore, initial distribution of the water saturation $s$ is known:

$$
s(x, 0)=\left\{\begin{array}{lll}
s_{0}^{\mathrm{p}} & \text { for } & x \in\left(0, x_{c}\right), \\
s_{0}^{\mathrm{f}} & \text { for } & x \in\left(x_{c}, 1\right) .
\end{array}\right.
$$




\subsubsection{Boundary conditions}

At the boundaries $x=0$ and $x=1$ we prescribe a no-flow condition, assuming that both paper and felt are in contact with impermeable press-rolls. This gives the Neumann conditions

$$
u_{x}(0, t)=u_{x}(1, t)=0 \text { for } 0<t<1 .
$$

\subsubsection{Cross conditions}

As in Part I, the mass and the pressure conservation yield

$$
\begin{aligned}
& \left.C_{\mathrm{w} 0}^{\mathrm{f}} g^{\mathrm{f}}(u) k_{\mathrm{w}}^{\mathrm{rf}}(s) u_{x}\right|_{\left(x_{c}^{+}, t\right)}=\left.C_{\mathrm{w} 0}^{\mathrm{p}} g^{\mathrm{p}}(u) k_{\mathrm{w}}^{\mathrm{rp}}(s) u_{x}\right|_{\left(x_{c}^{-}, t\right)}, \\
& \left.C_{\mathrm{a} 0}^{\mathrm{f}} \rho_{\mathrm{a} 0} \rho_{\mathrm{a}} g^{\mathrm{f}}(u) k_{\mathrm{a}}^{\mathrm{rf}}(s) u_{x}\right|_{\left(x_{c}^{+}, t\right)}=\left.C_{\mathrm{a} 0}^{\mathrm{p}} \rho_{\mathrm{a} 0} \rho_{\mathrm{a}} g^{\mathrm{p}}(u) k_{\mathrm{a}}^{\mathrm{rp}}(s) u_{x}\right|_{\left(x_{c}^{-}, t\right)}
\end{aligned}
$$

and

$$
p_{\mathrm{s} 0}^{\mathrm{f}}\left[\left.(u)^{q^{\mathrm{f}}}\right|_{\left(x_{c}^{+}, t\right)}-\left(\phi_{0}^{\mathrm{f}}\right)^{q^{\mathrm{f}}}\right]=p_{s 0}^{\mathrm{p}}\left[\left.(u)^{q^{\mathrm{p}}}\right|_{\left(x_{c}^{-}, t\right)}-\left(\phi_{0}^{\mathrm{p}}\right)^{q^{\mathrm{p}}}\right] .
$$

The continuity of $p_{\mathrm{a}}$ across the interface and (6) imply that also $\rho_{\mathrm{a}}$ is continuous. Thus $\rho_{\mathrm{a}}$ disappears from (14). The pressure condition implies that the void ratio $u$ is discontinuous across the paper-felt interface. Conditions (13) and (14) now give the jump in the saturation:

$$
\left.\frac{k_{\mathrm{w}}^{\mathrm{rf}}(s)}{k_{\mathrm{a}}^{\mathrm{rf}}(s)}\right|_{\left(x_{c}^{+}, t\right)}=\left.\frac{k_{\mathrm{w}}^{\mathrm{rp}}(s)}{k_{\mathrm{a}}^{\mathrm{rp}}(s)}\right|_{\left(x_{c}^{-}, t\right)} \text { for } 0<t<1 .
$$

\section{Investigation of Nature of System}

The model consists of the equations (8), (9) in the subdomains $Q^{\mathrm{p}}$ and $Q^{\mathrm{f}}$, subject to the initial conditions (10), (11), the boundary conditions (12), and the cross conditions (13), (14) and (15) across the paper-felt interface. As we will see, this system has similar mathematical features as the simplified system from Part I.

Since the following transformations apply to both the paper and felt domain we omit the subscript $i=\mathrm{p}$, f, to simplify notation. Dividing (9) by $\rho_{\mathrm{a}}(u, t)$ we obtain

$$
\begin{aligned}
& (u(1-s))_{t}+u(1-s) \frac{\left(\rho_{\mathrm{a}}(u, t)\right)_{t}}{\rho_{\mathrm{a}}(u, t)} \\
& \quad=\left(C_{\mathrm{a} 0} g(u) k_{\mathrm{a}}^{\mathrm{r}}(s) u_{x}\right)_{x}+C_{\mathrm{a} 0} g(u) k_{\mathrm{a}}^{\mathrm{r}}(s) u_{x} \frac{\left(\rho_{\mathrm{a}}(u, t)\right)_{x}}{\rho_{\mathrm{a}}(u, t)} .
\end{aligned}
$$

Differentiation of (7) gives

$$
\left(\rho_{\mathrm{a}}(u, t)\right)_{t}=\frac{1}{\gamma^{*}}\left(p_{\mathrm{T}}^{\prime}(t)-p_{\mathrm{s}}^{\prime}(u) u_{t}\right) \quad \text { and } \quad\left(\rho_{\mathrm{a}}(u, t)\right)_{x}=-\frac{1}{\gamma^{*}} p_{\mathrm{s}}^{\prime}(u) u_{x} .
$$


Thus adding (17) and (8) and dividing the obtained equation by

$$
A(u, s, t)=1-u(1-s) \frac{p_{\mathrm{s}}^{\prime}(u)}{\gamma^{*} \rho_{\mathrm{a}}(u, t)} \geqslant 1,
$$

we obtain an equation for $u$ :

$$
u_{t}=\frac{1}{A(u, s, t)}\left[\left(\left(C_{\mathrm{w} 0} k_{\mathrm{w}}^{\mathrm{r}}(s)+C_{\mathrm{a} 0} k_{\mathrm{a}}^{\mathrm{r}}(s)\right) g(u) u_{x}\right)_{x}+B\left(u, s, u_{x}, t\right)\right],
$$

where

$$
B\left(u, s, u_{x}, t\right)=-\frac{u(1-s) p_{\mathrm{T}}^{\prime}(t)+C_{\mathrm{a} 0} g(u) k_{\mathrm{a}}^{\mathrm{r}}(s) p_{\mathrm{s}}^{\prime}(u)\left(u_{x}\right)^{2}}{\gamma^{*} \rho_{\mathrm{a}}(u, t)} .
$$

Note that natural bounds for the void ratio $\left(0<u \geqslant u_{0}=\phi_{0}\right.$ : state of compression) and the saturation $(0<s<1)$ imply $A \geqslant 1$.

Writing

$$
s=S(u, r, t),
$$

where $r$ is a still unspecified function, and using

$$
s_{t}=S_{u} u_{t}+S_{\mathrm{r}} r_{\mathrm{t}}+S_{\mathrm{t}},
$$

equation (8) becomes

$$
\left(S+u S_{u}\right) u_{\mathrm{t}}+u S_{\mathrm{r}} r_{\mathrm{t}}+u S_{\mathrm{t}}=\left(C_{\mathrm{w} 0} g(u) k_{\mathrm{w}}^{\mathrm{r}}(S) u_{x}\right)_{x},
$$

and, with (18),

$$
\begin{aligned}
& \frac{S+u S_{u}}{A(u, S, t)}\left[\left(\left(C_{\mathrm{w} 0} k_{\mathrm{w}}^{\mathrm{r}}(S)+C_{\mathrm{a} 0} k_{\mathrm{a}}^{\mathrm{r}}(S)\right) g(u) u_{x}\right)_{x}+B\left(u, S, u_{x}, t\right)\right] \\
& \quad+u S_{\mathrm{r}} r_{\mathrm{t}}+u S_{\mathrm{t}}=\left(C_{\mathrm{w} 0} g(u) k_{\mathrm{w}}^{r}(S) u_{x}\right)_{x} .
\end{aligned}
$$

In order to eliminate $u_{x x}$ in the above equation we choose (19) such that

$$
\frac{S+u S_{u}}{A(u, S, t)}\left(C_{\mathrm{w} 0} k_{\mathrm{w}}^{\mathrm{r}}(S)+C_{\mathrm{a} 0} k_{\mathrm{a}}^{\mathrm{r}}(S)\right)=C_{\mathrm{w} 0} k_{\mathrm{w}}^{\mathrm{r}}(S) .
$$

Using (21) in (20) we obtain

$$
\begin{aligned}
& u S_{\mathrm{r}} r_{\mathrm{t}}+\left[F(S)\left(C_{\mathrm{w} 0} k_{\mathrm{w}}^{\mathrm{r}}(S)+C_{\mathrm{a} 0} k_{\mathrm{a}}^{\mathrm{r}}(S)\right)^{\prime}-\left(C_{\mathrm{w} 0} k_{\mathrm{w}}^{\mathrm{r}}(S)\right)^{\prime}\right] S_{x} g(u) u_{x} \\
& \quad=-B\left(u, S, u_{x}, t\right) F(S)-u S_{\mathrm{t}},
\end{aligned}
$$

for the fractional flow function $F(S)$ given by

$$
F(S)=\frac{m k_{\mathrm{w}}^{\mathrm{r}}(S)}{m k_{\mathrm{w}}^{\mathrm{r}}(S)+k_{\mathrm{a}}^{\mathrm{r}}(S)} .
$$


Here

$$
m=\frac{\mu_{\mathrm{a}}}{\mu_{\mathrm{w}}}=\frac{C_{\mathrm{w} 0}}{C_{\mathrm{a} 0}}
$$

is the air-water viscosity ratio. After elementary transformations we obtain an equation for $r$

$$
r_{\mathrm{t}}+\frac{f_{\mathrm{T}} F^{\prime}(S)}{u} r_{x}=-\frac{B\left(u, S, u_{x}, t\right)}{u S_{\mathrm{r}}} F(S)-\frac{f_{\mathrm{T}} F^{\prime}(S) S_{\mathrm{u}}}{u S_{\mathrm{r}}} u_{x}-\frac{S_{\mathrm{t}}}{S_{\mathrm{r}}},
$$

where the total (volume) flux is given as

$$
f_{\mathrm{T}}=-\left[C_{\mathrm{w} 0} k_{\mathrm{w}}^{\mathrm{r}}(S)+C_{\mathrm{a} 0} k_{\mathrm{a}}^{\mathrm{r}}(S)\right] g(u) u_{\mathrm{x}} .
$$

Using (19) we now have a coupled system in terms of $u$ and $r$ : for given $r$, the $u$-equation (18) is second order parabolic; for given $u$, the $r$-equation (22) is first order hyperbolic.

Due to technicalities involved, the details about the transformation (19) are moved to Appendix A.

From (22) it follows that the direction of characteristics for $r$ is

$$
\dot{x}(t)=\frac{f_{\mathrm{T}}}{u} \frac{\mathrm{d} F}{\mathrm{~d} S}(S) .
$$

Using the smoothness of transformation (19) it is possible to verify that $s$ has the same characteristic speed (23). No-flow conditions at $x=0$ and $x=1$ imply $f_{\mathrm{T}}=0$ and thus $\dot{x}(t)=0$, for all $t \in[0,1]$. Therefore no boundary conditions for $r$ are needed, since the corresponding characteristics at $x=0$ and $x=1$ do not enter the computational domain. The hyperbolic nature of the equation for $r$ explains also why only a single cross condition is required for this variable (and thus for $s$ ) at the paper-felt interface. It also explains why we may expect shocks for $r$. Since transformation (19) (more precisely, Problem $(T)$, see Appendix A) defines a smooth transformation, shocks in $r$ carry over to shocks in $s$. Using the definition of Problem $(T)$, an initial condition for $r$ can be derived, as in Part I.

As in Part I, we can show that if a shock in $s$ occurs at some curve $x=\zeta(t)$, then $u$ and the total flux $f_{\mathrm{T}}$ are continuous across the shock ( $u$ is only discontinuous across the interface $x=x_{\mathrm{c}}$ ). Interpreting equation (8) across the shock front yields a shock condition

$$
\frac{\mathrm{d} \zeta}{\mathrm{d} t}=\frac{1}{u(\zeta(t), t)} \frac{\left[f_{\mathrm{w}}\right]}{[s]},
$$

where $f_{\mathrm{w}}$ denotes the water flux

$$
f_{\mathrm{w}}=-C_{\mathrm{w} 0}^{\mathrm{f}} g(u) k_{\mathrm{w}}^{\mathrm{r}}(s) u_{\mathrm{x}},
$$

and [.] denotes jump, defined in Part I. 
Comparing to the model from Part I, where the boundary condition for $u$ at $x=1$ causes the flow, here the source term in $u$-equation acts as a driving force inside the domain. This is the fundamental difference between these two models.

For the sake of completeness, we consider briefly a single layer problem with no-flow boundary conditions. One easily verifies that constant in space functions $u=u(t)$ and $s=s(t)$ are the solutions of equations (8) and (9) and satisfy no-flow boundary conditions. Having in mind that material coordinates are used, this form of the solutions implies that there is no flow of fluids (water and air) relative to the solid particles. Furthermore, one can verify that $u(t)$ decreases as long as $p_{\mathrm{T}}(t)$ increases and vice versa. The physical interpretation is obvious: as long as the total applied pressure is increased, the medium is being compressed and inversely. In the case of two layers, due to cross conditions the solutions $u$ and $s$ depend on $x$ also.

\section{Computational Results}

We will first illustrate the main features of the solutions by a numerical example. In order to give a better qualitative description, we then take into account the plastic deformations of the paper. At the end, we address the problem of improving the efficiency of the press-nip using the proposed model.

\subsection{NUMERICAL METHOD}

To compute a numerical solution, a modification of the saturation-upwind method introduced in Part I is employed. An explicit discretization of the equations (8-9) is performed, using the central differences for $u_{x}$ and centered approximations for $u$ and $g(u)$. On the other side, $k_{j}^{\mathrm{r}}(s), j=\mathrm{w}$, a is approximated in an upwind manner (see (Bezanovic, 1995) for details).

Although we do not explicitly introduce the numerical scheme here, we will, in a less formal way, consider now its stability. Since the numerical scheme is based on system (8-9), we can not apply straightforwardly the criteria for the stability of parabolic and hyperbolic equations, giving the conditions for time step and grid size. Nevertheless, we will obtain a correct stability condition by considering the hyperbolic and parabolic parts of the system.

The stability criterion for one-dimensional hyperbolic equations is based on the fact that during one time step the information (characteristic) propagates not further than to a neighbouring cell, see (Leveque, 2002) for instance. The second condition follows the stability condition of the 
Table I. Parameter set used in computations

\begin{tabular}{|c|c|c|}
\hline \multicolumn{2}{|c|}{ parameter } & Value \\
\hline$p_{\text {atm }}$ & [MPa] & 0.1 \\
\hline$\rho_{\text {atm }}$ & {$\left[\mathrm{Kg} \mathrm{m}^{-3}\right]$} & 1.29 \\
\hline$\gamma$ & {$\left[\mathrm{MPa} \mathrm{Kg}^{-1} \mathrm{~m}^{3}\right]$} & $8.3 \times 10^{-2}$ \\
\hline
\end{tabular}

corresponding parabolic part (equation (18)), see (Hall and Porsching, 1990) for instance, implying that

$$
\frac{\Delta t}{(\Delta x)^{2}} \leqslant \frac{1}{2 \max _{Q^{\mathrm{p}} \cup Q^{\mathrm{f}}} \frac{C_{\mathrm{w}}+C_{\mathrm{a}}}{a}} .
$$

This is a dominant condition. If it is satisfied for some $\Delta t$ and $\Delta x$, we can verify (computing the characteristics speed (23)) that the above mentioned condition corresponding to the hyperbolic part is readily satisfied. The numerical experiments show that the condition (24) gives indeed a criterion for the stability of the numerical scheme.

\subsection{NUMERICAL EXAMPLE}

We consider now a numerical example. The parameters $u_{0}, s_{0}, k_{0}, p_{\mathrm{s} 0}, \lambda, q, h_{0}$ and $p_{\mathrm{T} 0}$ are the same as in Part I. The parameters for the perfect gas equation (1) are given in Table I.

The numerical results are given in Figures 1 and 2. For a better understanding of the results we can consider the time $t$ as a scaled horizontal (longitudinal) coordinate.

In this example, using the criterion from Part I, the initial shock is in the saturation $s$ is formed. This shock in $s$ propagates into the felt domain, the water and air flow from paper to felt. For $t \approx 0.16, s_{l}$ becomes larger then $s_{\text {in }}$ and the mentioned criterion from Part I for existence of shock in $s$ is not valid any more. This, shock like initial behaviour in $s$, caused by different initial saturations of paper and felt is reported also in (Kataja et al., 1992).

The fluid pressure and the saturation in paper increase in the first part of the press-nip and reach their maximum just before the mid-nip (middle of the nip). Consequently, in this region the water flow is the most intensive. These are well-known features of the wet paper pressing, see for instance (Nilson and Larson, 1968; Paulapuro, 2001). After the mid-nip (middle of the nip) the saturation decreases due to expansion of air in pores, but the flux at the paper-felt interface keeps the positive sign up to $t \approx 0.8$. After this moment, certain reverse flow, so-called rewetting, occurs. 

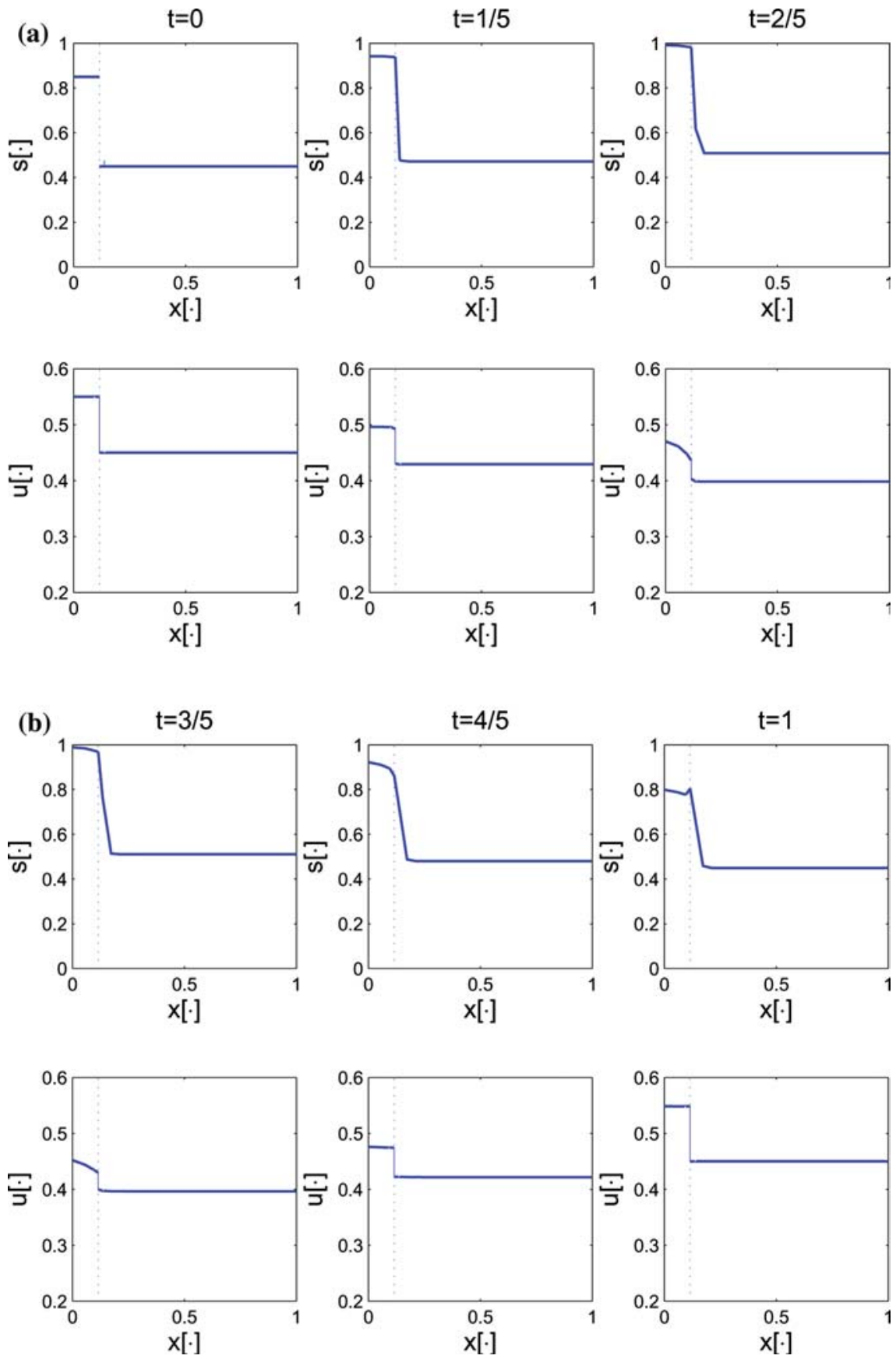

Figure 1. Example 2: distribution of $s$ and $u$ at different times. (a) $t=0,1 / 5$ and 2/5. (b) $t=3 / 5,4 / 5$ and 1 . 

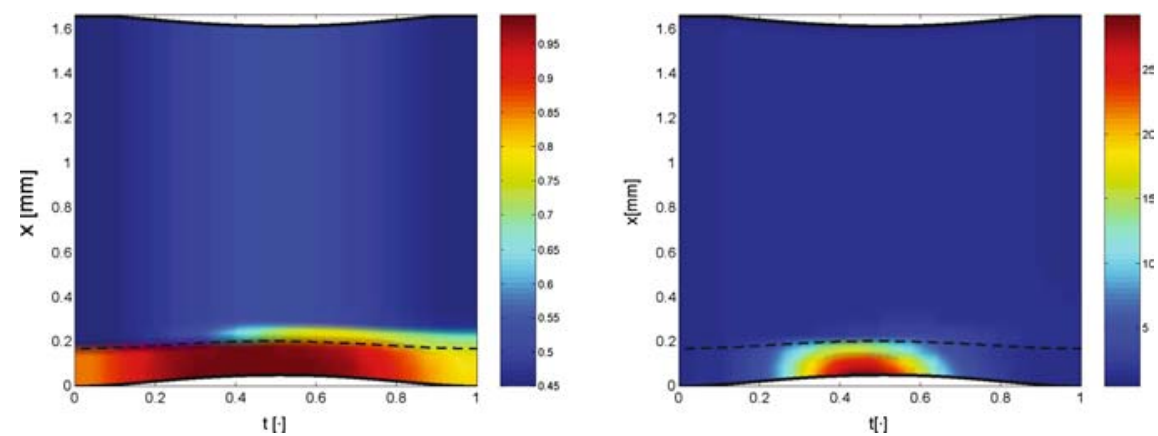

Figure 2. Example 2: saturation (a) and air density (b) in spatial coordinates.

This effects are also known, see (Nilson and Larson, 1968; Paulapuro, 2001) for instance.

To improve the behaviour of the model in expansion phase, in the next section we will take into account permanent deformation of the paper.

\subsection{PLASTIC DEFORMATION OF PAPER}

In the example from the previous section, the mechanical behaviour of fibrous network of paper was assumed to be perfectly elastic. This implies that absence of (solid) stress means absence of strain and that the stress-strain relationship is the same in compression and expansion phase. However, compressed wet paper can experience complicated permanent and semi-permanent deformations, see for instance (Nilson and Larson, 1968; Lobosco and Kaul, 2001). In other words, after releasing of pressure, paper does not regain (or at least not immediately) its original thickness. As reported by (El-Hosseiny, 1990), viscoelastic properties of the wet paper arise from the flow of fluids through the pores, while the pure fibrous network appears to be non-viscoelastic.

Therefore, we consider only plastic deformations of the wet paper web. This effect is modelled by assuming that the stress-strain (in fact, structural pressure-strain) relation is different for the compression and the expansion phase. Since strain is defined locally, in the expansion phase different particles inside the paper follow in general different stress-strain curves, see Figure 3. Using the relation (see Part I)

$$
\epsilon=u-u_{0},
$$

structural pressure-strain and structural pressure-void ratio relations directly imply each other, see Figure 3.

We consider only the numerical modelling of plastic effects here. We do not study the (non-trivial) mathematical implications when these different 


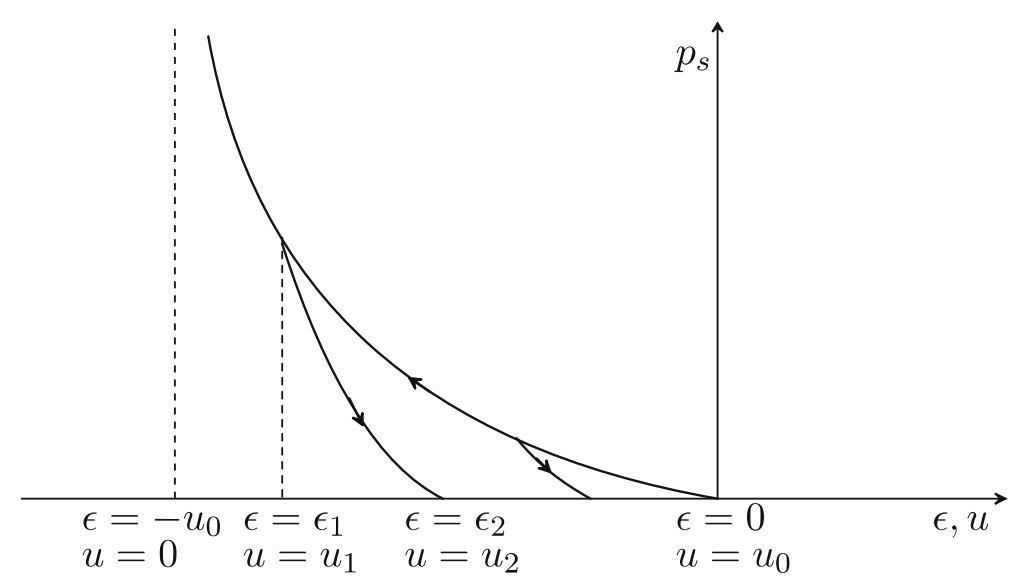

Figure 3. Pressure-strain (void ratio) curves for compression and expansion phase.

structural pressure-void ratio relations for compression and expansion used in the partial differential equations (8) and (9).

We will follow the idea from (Kataja et al., 1992), where the two-dimensional (transversal-longitudinal) model in stationary case is used to simulate the pressing of wet paper together with a rigid (incompressible) porous plate. In this study it is assumed that the particle which is deformed to maximal strain $\epsilon$, after releasing of the pressure returns to a strain $\epsilon_{\mathrm{p} 1} \epsilon$. Note that, in this interpretation, the absence of plastic deformations implies $\epsilon_{\mathrm{pl}}=0$.

In the compression phase the relation (3) is used. Let the value of void ratio of a certain particle in the moment when expansion starts be $u_{1}$, see Figure 3. The corresponding strain is $\epsilon_{1}=u_{1}-u_{0 \mathrm{p}}$. As we have mentioned, we assume that after the releasing of stress, i.e. for $p_{\mathrm{s}}=0$, the particle returns to state with strain equal to

$$
\epsilon_{2}=\epsilon_{\mathrm{p} l} \epsilon_{1}=\epsilon_{\mathrm{p} l}\left(u_{1}-u_{0 \mathrm{p}}\right)
$$

and the corresponding void ratio

$$
u_{2}=\epsilon_{2}+u_{0 \mathrm{p}}=\epsilon_{\mathrm{p} l} u_{1}+\left(1-\epsilon_{\mathrm{p} l}\right) u_{0 \mathrm{p}} .
$$

Taking, as in (Kataja et al., 1992), $\epsilon_{\mathrm{pl}}=1 / 2$ we have

$$
\epsilon_{2}=\frac{u_{1}-u_{0 \mathrm{p}}}{2} \quad \text { and } \quad u_{2}=\frac{u_{1}+u_{0 \mathrm{p}}}{2} .
$$

We take the structural pressure-void ratio relation in expansion phase as

$$
p_{\mathrm{s}}^{\exp }(u)=p_{\mathrm{s} 0}\left[u^{-q_{\exp }}-u_{2}^{-q_{\exp }}\right]
$$


i.e. with the same $p_{\mathrm{s} 0}$ as in (3). The parameter $q_{\exp }$ is chosen such that $p_{\mathrm{s}}^{\exp }\left(u_{1}\right)=p_{\mathrm{s}}\left(u_{1}\right)$.

In general, every particle has a different compression history and begins to expand in a different moment comparing to the other particles. However, due to no-flow boundary conditions, the gradient of void ratio (and consequently the gradient of strain) is not large (see Figure 1). For this reason we use the same relation (25) for all particles, from the moment when paper starts to expand.

Apart from relation (25), all others parameters are taken as in example from the previous section. In Figures 4(a) and 4(b) we show the evolution of paper thickness and the mass of water in paper, respectively, both for $\epsilon_{\mathrm{pl}}=0$ (no plastic effects) and $\epsilon_{\mathrm{p} l}=1 / 2$. We immediately observe the significant effects of permanent deformations on the paper thickness. Further, back flow (rewetting) occurs for $\epsilon_{\mathrm{p} l}=0$ and not for $\epsilon_{\mathrm{p} l}=1 / 2$. Therefore, in the case $\epsilon_{\mathrm{pl}}=1 / 2$, the water content in paper decreases during the whole nip. The expansion of paper occurs only due to expansion of air in the pores. Comparing the influence of plastic deformations on the solutions, (Kataja et al., 1992) reported very similar observations.

The effects of plastic deformations do not influence significantly on the quantity of the total water content in paper. However, they remarkable influence some other important paper properties: the total thickness and further mechanical behaviour.

There is a quantitative difference regarding the computed magnitude of water flow, comparing to (Kataja et al., 1992). The possible explanation could be that in this study the authors consider the incompressible porous plate. Therefore the compression of paper-plate is taken only by paper layer, implying a more intensive flow from paper to felt.
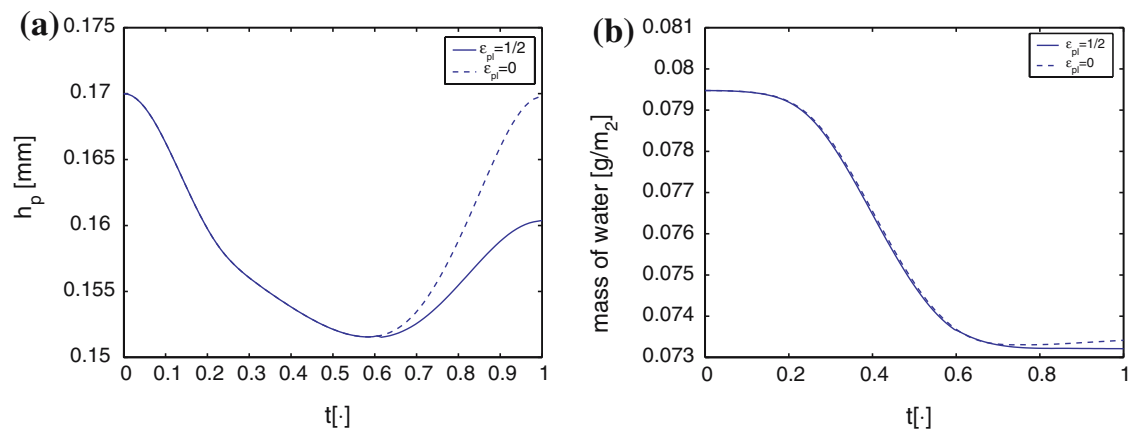

Figure 4. Time evolution of paper thickness (a) and the total mass of water in paper (b) for cases $\epsilon_{\mathrm{pl}}=1 / 2$ and $\epsilon_{\mathrm{p} l}=0$. 


\subsection{PERFORMANCE OF THE MODEL}

The often used quantity in wet paper pressing is the 'press impulse', i.e. the integral of the total pressure over the nip residence time, see (Back, 1998). This is a measure of the energy consumed by a press-nip. An important problem is to consider which press regime has the best efficiency for a fixed energy, i.e. to consider is which type of press pulses (which shape of the total pressure curve) for the same impulse produces best dewatering. This is correlated with the choice of the size of the press rolls, which consequently exert different pressure curves (for the same press impulse, the smaller roll exerts higher maximal pressure but has a smaller nip residence time). We do not consider here the corresponding influences on paper quality.

We employ the proposed model to answer this question and consider three cases with different press-nip time and the maximal applied pressure. For $i=1,2,3$ we take

$$
p_{\mathrm{T} i}(t)=\alpha_{i} p_{\mathrm{T} 0} \sin ^{2}(\pi t) \quad \text { and } \quad t_{\mathrm{fin}}=t_{\mathrm{fin} 0} / \alpha_{i},
$$

Here $p_{\mathrm{T} 0}=5 \mathrm{MPa}, t_{\text {fin } 0}=2.4 \times 10^{-2} \mathrm{~s}, \alpha_{1}=3 / 2, \alpha_{2}=1$ and $\alpha_{3}=2 / 3$, see Figure 5(a). Note that in all three case the press impulses are equal:

$$
\int_{0}^{t_{\mathrm{fin}}} p_{\mathrm{T} i}(t) d t=\frac{1}{2} p_{\mathrm{T} 0} t_{\mathrm{fin}}, \quad i=1,2,3 .
$$

Taking into account plastic effects (with $\epsilon_{\mathrm{pl}}=0.5$ ) and computing the evolution of total mass of water in paper (Figure 5(b)) we conclude that harder pressure pulses (with higher maximal pressure) produce better drying results. This conclusion is in agreement with earlier observations, see (Back, 1998) for instance.

The experiments with different felt permeabilities $k_{0}^{\mathrm{f}}$ suggest that, as expected, a larger felt permeability improves the drying results.
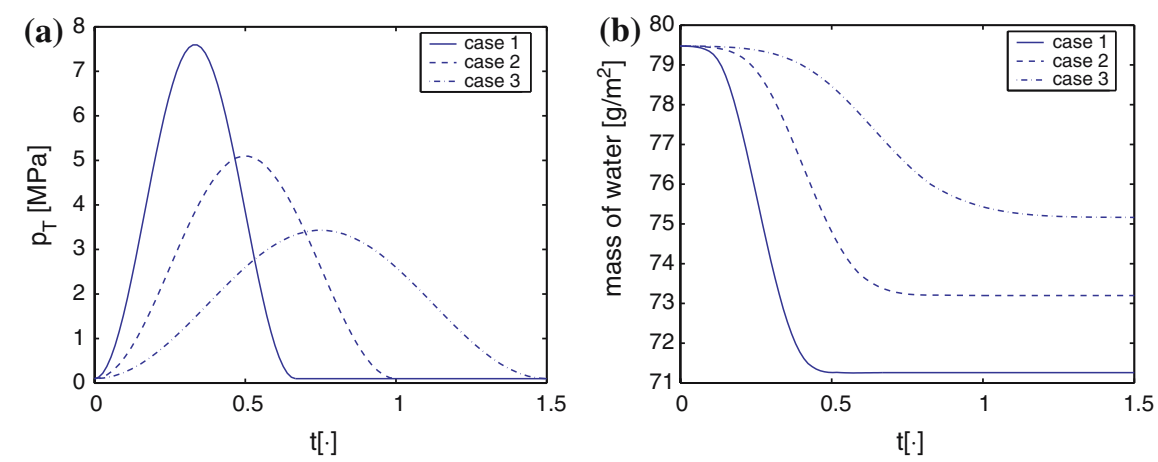

Figure 5. Three cases of different press pulses (a) and corresponding (time) evolution of relative paper dryness (b). 


\section{Conclusions}

In this paper we have studied a three-phase model for wet pressing of paper for the compressible air case. We have introduced a suitable transformation in order to rewrite the model into the standard parabolichyperbolic form. Employing a suitable upwind method, we have performed the computations, including also the effects of plastic deformations.

The comparisons with experimental results show that the proposed model, although one-dimensional, gives a good qualitative description of the wet pressing process. At the other hand, the horizontal air flow, which seems to be of importance in the beginning and in the end of nip, is not easy to describe using the proposed one-dimensional transversal model. Therefore a further step to improve the results could be to consider a two-dimensional model.

In order to compare quantitative output of the model (calculated dryness gain for instance) with some experimentally obtained values, we need to adjust values of all parameters to the specific case. We welcome contributions to this validation process.

\section{Acknowledgements}

This work was supported by the Netherlands Organization for Scientific Research (NWO), through the project 613.002.046. The authors are grateful to Sorin Pop (Department of Mathematics and Computer Science, Eindhoven University of Technology) for critical reading of this paper.

\section{Appendix: A. Transformation (19)}

To satisfy (21) we define (19) as the solution of the backward initial value problem

$$
(T):\left\{\begin{array}{l}
\frac{\mathrm{d} S}{\mathrm{~d} u}=\frac{1}{u}(F(S) A(u, S, t)-S)=Q(u, S, t) \text { for } 0<u<u_{0}, \\
S\left(u_{0}\right)=r .
\end{array}\right.
$$

Taking $v=1 / u$, problem $(T)$ transforms into a forward initial value problem

$$
\left(T^{v}\right):\left\{\begin{array}{l}
\frac{\mathrm{d} S}{\mathrm{~d} v}=\frac{1}{v}(S-F(S) A(1 / v, S, t))=Q^{v}(v, S, t) \text { for } v_{0}<v<\infty, \\
S\left(v_{0}\right)=r,
\end{array}\right.
$$

where $v_{0}=1 / u_{0}$. The smoothness of $Q^{v}$ implies the existence of the solution $S^{v}(v, r, t)$ of Problem $\left(T^{v}\right)$, see (Coddington and Levinson, 1995, Theorem 1.2 , p. 6)) of Problem (T). By (Coddington and Levinson, 1955, Theorem 7.2, p. 25) and (Coddington and Levinson, 1955, Theorem 7.5, p. 30) 


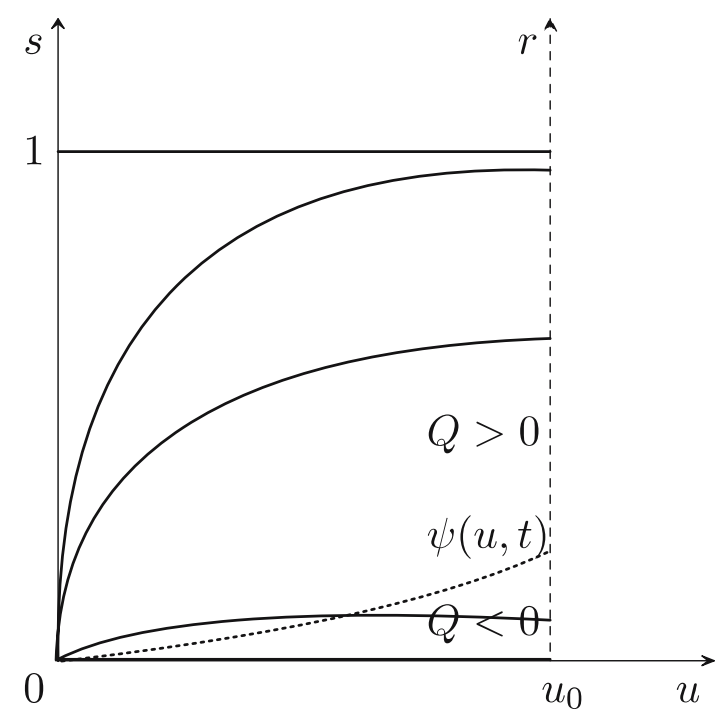

Figure 6. Sketch of solutions of Problem $(T)$ in $(u, S)$ space plane.

it follows that $S^{v}$ is continuously differentiable in $v$ and $r$, as well as in the parameter $t$. Hence, Problem $(T)$ has a solution $S(u, r, t)=S^{v}(1 / u, r, t)$ which is smooth in $u, r$ and $t$.

Now we fix $t \in[0,1]$ and, to simplify consideration, we restrict to the special case of Brooks-Corey relative permeabilities (5). For any $\hat{u} \in\left(0, u_{0}\right]$ we have $Q(\hat{u}, 0, t)=0$ and $Q_{\mathrm{s}}(\hat{u}, 0, t)=-1 / \hat{u}<0$, whereas $Q(\hat{u}, 1, t)=0$ and $Q_{\mathrm{s}}(\hat{u}, 1, t)=\left(\hat{u} p_{\mathrm{s}}^{\prime}(\hat{u}) /\left(\gamma^{*} \rho_{\mathrm{a}}(\hat{u}, t)\right)-1\right) / \hat{u}<0$. Therefore there exists $\hat{s} \in(0,1)$ such that $Q(\hat{u}, \hat{s}, t)=0$. In this specific setting (Brooks-Corey relative permeabilities), the value $\hat{s}$ is unique for any given $\hat{u}$. In this way we obtain a curve $S=\psi(u, t)$ for $u \in\left(0, u_{0}\right]$, defined by $Q(u, \psi(u, t), t)=0$ (the dotted curve in Figure 6). Note that $\psi$ is continuous (by the continuity of $Q$ ), and

$$
Q:\left\{\begin{array}{ccc}
<0 & \text { for } & 0<S<\psi(u, t) \\
>0 & \text { for } & \psi(u, t)<S<1
\end{array}\right.
$$

Further, since

$$
Q=\frac{1}{u}\left[F(S)\left(1+(1-s) \frac{p_{\mathrm{s} 0} q u^{-q}}{k \rho_{\mathrm{a}}(u, t)}\right)-S\right],
$$

and because $1 / u, 1 / \rho_{\mathrm{a}}(u, t)$ and $u^{-q}$ are decreasing in $u, Q$ is decreasing in $u$. This implies that curve $\psi(u, t)$ is increasing in $u$ (i.e. $\psi$ decreases with decreasing $u$ ). Therefore there exists $\lim _{u \downarrow 0} \psi(u, t)$. This limit must be equal to 0 , since all solutions of Problem $(T)$ end up at the equilibrium value $S=0$ for $u \downarrow 0$, see Figure 6 . 
For fixed $t \in[0,1]$ and $u \in\left(0, u_{0}\right), s=S(u, r, t)$ defines a one-to-one correspondence between $s$ and $r$. This is a direct consequence of uniqueness for problem $(T)$ (orbits cannot intersect).

\section{References}

Bear, J.: 1972, Dynamics of Fluids in Porous Media, Elsevier, New York.

Back, E. L.: 1998, Steam boxes in press sections-possibilities and limitations, Appita 41(3), 217-223.

Bežanović, D.: 2005, Mathematical Modelling of Wet Paper Pressing. PhD thesis, Eindhoven University of Technology, 2005.

Bežanović, D., Duijn, C. J. van and Kaasschieter, E. F.: 2006, Analysis of paper pressing: the saturated one-dimensional case. J. Appl. Math. Mech. 86(1), 18-36.

Bežanović, D., van Duijn, C. J. and Kaasschieter, E. F.: 2007, Analysis of wet pressing of paper: the three-phase model. Part I: constant air density, Report CASA 05-16 of the Department of Mathematics and Computer Science, Eindhoven, University of Technology. In press.

Coddington, E. A. and Levinson, N.: 1955, Theory of Ordinary Differential Equations, McGraw Hill.

El-Hosseiny, F.: 1990, Nordic Pulp and Paper Research Journal 1, 28.

Hall, C. A. and Porsching, T. A.: 1990, Numerical Analysis of Partial Differential Equations, Englewood Cliffs.

Helmig, R.: 1997, Multiphase Flow and Transport Processes in the Subsurface, SpringerVerlag, Berlin.

Kataja, M., Hiltunen, K. and Timonen, J.: 1992, Flow of water and air in a compressible porous medium. A model of wet pressing of paper, J. Phys. D: Appl. Phys. 25, 1053-1063.

Leveque, R. J.: 2002, Finite Volume Methods for Hyperbolic Problems, Cambridge university press.

Lobosco, V. and Kaul, V.: 2001, An elastic-viscoplastic model of the fibre network stress in wet pressing: Part I, Nordic Pulp Paper Res. J. 16(1), 12-17.

Nilson, P and Larson, K. O.: 1968, Paper web performance in a press nip, Pulp Paper Mag. Canada December 20, 66-73.

Paulapuro, H.: 2001, Wet pressing-present understanding and future challenges, 12th Fundamental Research Symposium, Oxford. 